\title{
EDITORIAL
}

\section{Fibrocytes: potential new therapeutic targets for pulmonary hypertension?}

\author{
K.R. Stenmark, M.G. Frid and M.E. Yeager
}

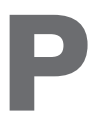

ulmonary arterial hypertension $(\mathrm{PAH})$ is a progressive fatal disease [1, 2]. All forms of chronic pulmonary hypertension are characterised by cellular and structural changes in the walls of the pulmonary arteries. Intimal thickening and fibrosis, medial hypertrophy and fibroproliferative changes in the adventitia are common [3]. Virtually all of these changes are characterised, to a greater or lesser degree, by increased numbers of cells expressing smooth muscle $\alpha$ actin $(\alpha-S M A)$, as well as the accumulation of inflammatory cells [4-7]. At present, neither the origin of the accumulating cells, particularly those expressing $\alpha$-SMA, nor the molecular mechanisms operating to cause their accumulation has been fully delineated. Traditionally, it has been thought that the $\alpha$ SMA-expressing and/or collagen-producing cells accumulating in vascular lesions were exclusively derived from resident vascular cells. However, since the late 1990s, this concept has been extended by the fact that bone-marrow-derived circulating progenitor cells are recruited to sites of vascular injury and contribute to both the vascular repair and pathological remodelling by differentiating into cells expressing mesenchymal or even smooth-muscle-like characteristics [8-10].

Among the many populations of progenitor cells that may be recruited to the vessel wall and assume mesenchymal cell characteristics are fibrocytes. These cells were initially described by BucALA et al. [11] as circulating bone-marrowderived cells with the ability to adapt a mesenchymal phenotype. They share certain features with both fibroblasts and monocytes, and this combination of connective tissue cell and myeloid cell characteristics permits their identification by a number of markers. Fibrocytes express the stem cell marker CD34, the pan-haematopoietic marker CD45 and monocyte markers, such as CD14 and CD11, and produce components of the connective tissue matrix, including collagen I, collagen III and vimentin $[12,13]$. Fibrocytes display many properties that are important for wound repair. Upon entry into diseased tissues, fibrocytes are believed to adapt the phenotype of a myofibroblast, as evidenced by acquisition of $\alpha$-SMA expression [12, 14, 15]. However, the extent to which fibrocytes transition into $\alpha$-SMA-expressing cells in different settings of tissue repair and injury remains unclear and may be

Cardiovascular Pulmonary Research Laboratory, Depts of Pediatrics and Medicine, University of Colorado Denver, Aurora, CO, USA.

CORRESPONDENCE: K.R. Stenmark, University of Colorado Denver, 12700 E. 19th Avenue, Research 2, Box 131, Aurora, C0 80045, USA. E-mail: kurt.stenmark@ucdenver.edu exquisitely injury- and/or organ-specific. In addition, fibrocytes display other properties that are important for wound repair. They secrete pro-inflammatory cytokines, including tumour necrosis factor (TNF), macrophage inflammatory protein (MIP) $-1 \alpha / \beta$ and interleukin (IL)- $6,-8$ and -10 , as well as matrix metalloproteinase- 2 and -9 . In addition, fibrocytes can exhibit a pro-angiogenic phenotype both in vitro and in vivo [16]. Fibrocytes also express a number of chemokine receptors, including CXC chemokine receptor (CXCR) 4 and CC chemokine receptor (CCR) 7, which regulate their recruitment and trafficking $[17,18]$. Furthermore, fibrocytes express major histocompatibility complex class II and demonstrate antigen-presenting capabilities in vitro and in vivo $[16,19]$.

Based on these properties, it is not unexpected that fibrocytes or related circulating mesenchymal precursor cells have been shown to participate in both systemic and pulmonary vascular remodelling processes. In the systemic circulation, fibrocytes have been identified in the fibrous cap of human atherosclerotic lesions [20, 21]. Further, one of the subsets of monocytes that are preferentially recruited into the arterial wall during experimentally induced arteriosclerosis in mice represents the murine counterpart of the human inflammatory subset of CD14+/CD16- mononuclear cells that express the CC chemokine ligand (CCL) 2/monocyte chemoattractant protein (MCP)-1 receptor CCR2 [22]. Consistent with findings from the murine models are studies demonstrating that the intimal hyperplasia in an ovine carotid artery patch graft model is partially due to haematopoietic circulating fibrocytes that acquire mesenchymal features as they mature at the site of injury [23]. Other studies demonstrate that fibrocytes or fibrocyte-like cells (CD14+-derived mesenchymal cells) play an important role in the remodelling that characterises transplant vasculopathy [24-27]. Importantly, these observations in the systemic circulation are supported by epidemiological data showing that CCL2/MCP-1 plays a major role in the pathogenesis of atherosclerosis and may promote recruitment of fibrocytes (known to express CCR2 and CCR7) to the vessel wall [28]. In the pulmonary circulation, a critical contribution of fibrocytes to vascular remodelling has been described in the large mammalian model (neonatal calf), and in the rat model of chronic-hypoxia-induced pulmonary hypertension [29]. In the latter (rat) model, it has been shown that sustained hypoxia leads to the development of a complex pulmonary-artery-specific pro-inflammatory microenvironment that promotes recruitment, retention and differentiation of circulating fibrocytes to the pulmonary circulation [30]. 
The study of NIKAM et al. [31] in the current issue of the European Respiratory Journal provides further support that fibrocytes accumulate in the pulmonary arteries of animals with chronic hypoxic pulmonary hypertension. This work builds on previous studies demonstrating that, in both a large animal model (neonatal calf) and hypoxic rats and mice, fibrocytes accumulate in the perivascular lesions of the vessel wall under chronically hypoxic conditions [29, 32]. Accumulations of other mesenchymal progenitor cells have also been described in the hypoxic vessel wall and are characterised by the expression of mast/ stem cell growth factor receptor [33, 34]. More importantly, previous studies have demonstrated a causal link between fibrocyte accumulation and vascular wall remodelling by showing that depletion of the circulating population of mononuclear phagocytic cells, comprising among others the fibrocyte population, abrogate pulmonary perivascular remodelling in the rat model of hypoxic pulmonary hypertension [29]. These observations are consistent with observations of fibrotic remodelling in a variety of other organs [35], where cells that express the combination of CD45 and procollagen I have been consistently observed. However, since recent studies [13, 36] demonstrate some overlap in the markers used to identify fibrocytes and other monocyte-derived collagen-expressing cells, it should be considered that there is a range of monocytic cell subpopulations, which may best be described as mononuclear phagocytic cells, capable of expressing mesenchymal markers and contributing to vascular/fibrotic remodelling. Collectively, the results identify bone-marrow-derived mesenchymal precursors as important contributors to the vascular pathology that characterises pulmonary hypertension. It is thus clear that strategies aimed at the inhibition of recruitment or function of these cells could be important as a treatment for the disease process itself, a possibility explored in the study of NiKAM et al. [31].

Prostacyclin $\left(\mathrm{PGI}_{2}\right)$ is a commonly used agent for the treatment of severe pulmonary hypertension. The initial rationale for its use was based largely on its ability to act as a potent vasodilator and vascular protective agent. It has been shown to improve symptoms and survival in patients with idiopathic $\mathrm{PAH}$, as well as scleroderma-associated PAH [37, 38]. NIKAM et al. [31] examined the possibility that the stable $\mathrm{PGI}_{2}$ analogue treprostinil would attenuate pulmonary hypertension and vascular remodelling by inhibiting the recruitment of fibrocytes to the vessel wall and/or their differentiation. Intriguingly, they found that treprostinil treatment exhibited moderate but significant inhibition of remodelling and a reduction in the number of fibrocytes recruited to the hypoxic vessel wall. The mechanisms responsible for these effects were not explored in the manuscript, but they are perhaps not entirely surprising. Recent studies have demonstrated that $\mathrm{PGI}_{2}$ exhibits significant anti-inflammatory actions. For instance, $\mathrm{PGI}_{2}$ inhalation suppressed asthma in the mouse ovalbumin-induced model by reducing both the migration of dendritic cells to sites of inflammation and the type-2 T-helper cell (Th2) response (reduced IL-4, -5 and -13) [39]. Independent studies corroborated these findings by demonstrating that iloprost suppresses CCL17, CCL11, lung eosinophil accumulation and the recruitment of CD4+ Th2 to the airways in asthma models $[40,41]$. $\mathrm{PGI}_{2}$ counteracts the capacity of T-cells to produce the pro-inflammatory cytokines IL-12, p70, TNF- $\alpha$,
MIP-1 $\alpha$, MCP-1 and IL-6 [42, 43]. PGI 2 has also been demonstrated to reduce lipopolysaccharide-induced cytokine production in leukocytes through the inhibition of nuclear factor- $\kappa \mathrm{B}[44]$. $\mathrm{PGI}_{2}$ has also been demonstrated to exert antiinflammatory capabilities in vascular diseases. In patients with type-2 diabetes, $\mathrm{PGI}_{2}$ reduced circulating levels of the vascular inflammatory marker vascular cell adhesion molecule-1, as well as arterial intimal thickness [45]. In the setting of $\mathrm{PAH}$, $\mathrm{PGI}_{2}$ therapy has been demonstrated to clearly reduce the increased levels of MCP-1 found in these patients [46]. Moreover, a recent study demonstrates insight into the mechanism(s) whereby $\mathrm{PGI}_{2}$ inhibits cytokine production by monocytes by showing that $\mathrm{PGI}_{2}$ limits pro-inflammatory cytokine production by reducing transactivation-domaindependent recruitment of histone acetylase cAMP response element-binding protein-binding protein to transcription factors driving pro-inflammatory gene transcription [47]

The fact that $\mathrm{PGI}_{2}$ did not provide complete protection against hypoxia-induced remodelling is perhaps not unexpected. As stated, several chemokines and signalling pathways have been implicated in fibrocyte recruitment and differentiation within tissue sites. It has been demonstrated that fibrocyte differentiation from CD14+ monocytic precursors is augmented by the Th2 cytokines IL- 4 and IL-13, and is inhibited by the Th1 cytokines interferon- $\gamma$, TNF and IL-10 [48]. Further, fibrocytes are capable of producing a number of cytokines and growth factors that could cause resident cells to undergo hypertrophy, proliferate or recruit other inflammatory cells. Thus alternative and/or combinatorial therapies to $\mathrm{PGI}_{2}$ would seemingly be necessary for more complete prevention and probably for regression of established vascular lesions. Signalling pathways implicated in fibrocyte growth have included immunoreceptor tyrosine kinase motifs, mammalian target of rapamycin (mTOR), phosphatidylinositol-3'-kinase and angiotensin II receptor. Treatment of fibrocyte precursors with the Fc receptor antagonist serum amyloid $\mathrm{P}$, the $\mathrm{mTOR}$ inhibitor rapamycin or the angiotensin II receptor antagonist valsartan has been demonstrated to attenuate fibrocyte accumulation in mouse models of fibrosis $[49,50]$. Serum amyloid $P$ has shown encouraging results in pre-clinical studies of lung, heart and renal fibrosis, and in a phase-I clinical trial for the prevention of corneal scarring. Interventions that target the receptors and chemokines mediating fibrocyte trafficking and accumulation, such as MCP-1/CCL2 and stromal-cell-derived factor-1/CXC chemokine ligand (CXCL) 12, may also be beneficial in selected circumstances [49]. The latter is especially intriguing as a number of studies have shown that targeting the CXCL12/ CXCR4 axis blocks the pulmonary hypertensive response induced by hypoxia $[1,2,34]$.

Thus, given the fact that most currently available therapeutic options for pulmonary hypertension have not shown dramatic success in reducing the remodelling process, it is becoming increasingly clear that fibrocyte-directed therapies may need to target not one but several checkpoints, which would include the trafficking, differentiation and function of these cells.

The current study compels consideration of combinatorial approaches to research and treatment aimed at improving vasodilation, decreasing vascular remodelling and extending the ability to therapeutically modulate fibrocyte biology. 


\section{STATEMENT OF INTEREST}

None declared.

\section{REFERENCES}

1 Humbert M, Sitbon O, Chaouat A, et al. Survival in patients with idiopathic, familial, and anorexigen-associated pulmonary arterial hypertension in the modern management era. Circulation 2010; 122: 156-163.

2 Humbert M, Sitbon O, Yaïci A, et al. Survival in incident and prevalent cohorts of patients with pulmonary arterial hypertension. Eur Respir J 2010; 36: 549-555.

3 Morrell NW, Adnot S, Archer SL, et al. Cellular and molecular basis of pulmonary arterial hypertension. J Am Coll Cardiol 2009; 54: Suppl. S20-S31.

4 Humbert M, Morrell NW, Archer SL, et al. Cellular and molecular pathobiology of pulmonary arterial hypertension. J Am Coll Cardiol 2004; 43: Suppl. S, 13S-24S.

5 Majka SM, Skokan M, Wheeler L, et al. Evidence for cell fusion is absent in vascular lesions associated with pulmonary arterial hypertension. Am J Physiol 2008; 295: L1028-L1039.

6 Pietra GG, Capron F, Stewart S, et al. Pathologic assessment of vasculopathies in pulmonary hypertension. J Am Coll Cardi 2004; 43: Suppl. S, 25S-32S.

7 Yi ES, Kim H, Ahn H, et al. Distribution of obstructive intimal lesions and their cellular phenotypes in chronic pulmonary hypertension. A morphometric and immunohistochemical study. Am J Respir Crit Care Med 2000; 162: 1577-1586.

8 Iwata H, Sata M. Potential contribution of bone marrow-derived precursors to vascular repair and lesion formation: lessons from animal models of vascular diseases. Front Biosci 2007; 12: 4157-4167.

9 Tanaka K, Sata M. Contribution of circulating vascular progenitors in lesion formation and vascular healing: lessons from animal models. Current Opin Lipidol 2008; 19: 498-504.

10 Bellini A, Mattoli S. The role of the fibrocyte, a bone marrowderived mesenchymal progenitor, in reactive and reparative fibroses. Lab Invest 2007; 87: 858-870.

11 Bucala R, Spiegel LA, Chesney J, et al. Circulating fibrocytes define a new leukocyte subpopulation that mediates tissue repair. Mol Med 1994; 1: 71-81.

12 Herzog EL, Bucala R. Fibrocytes in health and disease. Exp Hematol 2010; 38: 548-556.

13 Pilling D, Fan T, Huang D, et al. Identification of markers that distinguish monocyte-derived fibrocytes from monocytes, macrophages, and fibroblasts. PLoS One 2009; 4: e7475.

14 Abe R, Donnelly SC, Peng T, et al. Peripheral blood fibrocytes: differentiation pathway and migration to wound sites. J Immunol 2001; 166: 7556-7562.

15 Schmidt M, Sun G, Stacey MA, et al. Identification of circulating fibrocytes as precursors of bronchial myofibroblasts in asthma. J Immunol 2003; 171: 380-389.

16 Hartlapp I, Abe R, Saeed RW, et al. Fibrocytes induce an angiogenic phenotype in cultured endothelial cells and promote angiogenesis in vivo. FASEB J 2001; 15: 2215-2224.

17 Gomperts BN, Strieter RM. Fibrocytes in lung disease. J Leukoc Biol 2007; 82: 449-456.

18 Phillips RJ, Burdick MD, Hong $\mathrm{K}$, et al. Circulating fibrocytes traffic to the lungs in response to CXCL12 and mediate fibrosis. J Clin Invest 2004; 114: 438-446.

19 Chesney J, Metz C, Stavitsky AB, et al. Regulated production of type I collagen and inflammatory cytokines by peripheral blood fibrocytes. J Immunol 1998; 160: 419-425.

20 Medbury H. Role of fibrocytes in atherogenesis. In: Bucala R, ed. Fibrocytes: New Insights into Tissue Repair and Systemic Fibroses. Singapore, World Scientific Publishing Co., 2007; pp. 175-194.
21 Medbury HJ, Tarran SL, Guiffre AK, et al. Monocytes contribute to the atherosclerotic cap by transformation into fibrocytes. Int Angiol 2008; 27: 114-123.

22 Tacke F, Randolph GJ. Migratory fate and differentiation of blood monocyte subsets. Immunobiology 2006; 211: 609-618.

23 Varcoe RL, Mikhail M, Guiffre AK, et al. The role of the fibrocyte in intimal hyperplasia. J Thromb Haemost 2006; 4: 1125-1133.

24 Hillebrands JL, Onuta G, Rozing J. Role of progenitor cells in transplant arteriosclerosis. Trends Cardiovasc Med 2005; 15: 1-8.

25 Onuta G, van Ark J, Rienstra H, et al. Development of transplant vasculopathy in aortic allografts correlates with neointimal smooth muscle cell proliferative capacity and fibrocyte frequency. Atherosclerosis 2010; 209: 393-402.

26 Sugiyama S, Kugiyama K, Nakamura S, et al. Characterization of smooth muscle-like cells in circulating human peripheral blood. Atherosclerosis 2006; 187: 351-362.

27 Hillebrands JL, Klatter FA, van den Hurk BM, et al. Origin of neointimal endothelium and $\alpha$-actin-positive smooth muscle cells in transplant arteriosclerosis. J Clin Invest 2001; 107: 1411-1422.

28 McDermott DH, Yang Q, Kathiresan S, et al. CCL2 polymorphisms are associated with serum monocyte chemoattractant protein-1 levels and myocardial infarction in the Framingham Heart Study. Circulation 2005; 112: 1113-1120.

29 Frid MG, Brunetti JA, Burke DL, et al. Hypoxia-induced pulmonary vascular remodeling requires recruitment of circulating mesenchymal precursors of a monocyte/macrophage lineage. Am J Pathol 2006; 168: 659-669.

30 Burke DL, Frid MG, Kunrath CL, et al. Sustained hypoxia promotes the development of a pulmonary artery-specific chronic inflammatory microenvironment. Am J Physiol 2009; 297: L238-L250.

31 Nikam VS, Schermuly RT, Dumitrascu R, et al. Treprostinil inhibits the recruitment of bone marrow-derived circulating fibrocytes in chronic hypoxic pulmonary hypertension. Eur Respir J 2010; 36: 1302-1314.

32 Hayashida K, Fujita J, Miyake $Y$, et al. Bone marrow-derived cells contribute to pulmonary vascular remodeling in hypoxia-induced pulmonary hypertension. Chest 2005; 127: 1793-1798.

33 Davie NJ, Crossno JT Jr, Frid MG, et al. Hypoxia-induced pulmonary artery adventitial remodeling and neovascularization: contribution of progenitor cells. Am J Physiol 2004; 286: L668-L678.

34 Young KC, Torres E, Hatzistergos KE, et al. Inhibition of the SDF-1/CXCR4 axis attenuates neonatal hypoxia-induced pulmonary hypertension. Circ Res 2009; 104: 1293-1301.

35 Sakai N, Wada T, Yokoyama H, et al. Secondary lymphoid tissue chemokine (SLC/CCL21)/CCR7 signaling regulates fibrocytes in renal fibrosis. Proc Natl Acad Sci USA 2006; 103: 14098-14103.

36 Schnoor M, Cullen P, Lorkowski J, et al. Production of type VI collagen by human macrophages: a new dimension in macrophage functional heterogeneity. J Immunol 2008; 180: 5707-5719.

37 Gomberg-Maitland M, Olschewski H. Prostacyclin therapies for the treatment of pulmonary arterial hypertension. Eur Respir J 2008; 31: 891-901.

38 Badesch DB, Tapson VF, McGoon MD, et al. Continuous intravenous epoprostenol for pulmonary hypertension due to the scleroderma spectrum of disease. A randomized, controlled trial. Ann Intern Med 2000; 132: 425-434.

39 Idzko M, Hammad $\mathrm{H}$, van Nimwegen $\mathrm{M}$, et al. Inhaled iloprost suppresses the cardinal features of asthma via inhibition of airway dendritic cell function. J Clin Invest 2007; 117: 464-472.

40 Jaffar Z, Ferrini ME, Buford MC, et al. Prostaglandin $\mathrm{I}_{2}$-IP signaling blocks allergic pulmonary inflammation by preventing recruitment of CD4+ Th2 cells into the airways in a mouse model of asthma. J Immunol 2007; 179: 6193-6203.

41 Jaffar Z, Wan KS, Roberts K. A key role for prostaglandin $I_{2}$ in limiting lung mucosal Th2, but not Th1, responses to inhaled allergen. J Immunol 2002; 169: 5997-6004.

42 Zhou W, Hashimoto K, Goleniewska $\mathrm{K}$, et al. Prostaglandin $\mathrm{I}_{2}$ analogs inhibit proinflammatory cytokine production and $\mathrm{T}$ cell 
stimulatory function of dendritic cells. J Immunol 2007; 178 702-710.

43 Zhou W, Blackwell TS, Goleniewska $\mathrm{K}$, et al. Prostaglandin $\mathrm{I}_{2}$ analogs inhibit Th1 and Th2 effector cytokine production by CD4 T cells. J Leukoc Biol 2007; 81: 809-817.

44 Raychaudhuri B, Malur A, Bonfield TL, et al. The prostacyclin analogue treprostinil blocks NFKB nuclear translocation in human alveolar macrophages. J Biol Chem 2002; 277: 33344-33348.

45 Goya $\mathrm{K}$, Otsuki $\mathrm{M}, \mathrm{Xu}$ X, et al. Effects of the prostaglandin $\mathrm{I}_{2}$ analogue, beraprost sodium, on vascular cell adhesion molecule-1 expression in human vascular endothelial cells and circulating vascular cell adhesion molecule-1 level in patients with type 2 diabetes mellitus. Metabolism 2003; 52: 192-198.

46 Katsushi H, Kazufumi N, Hideki F, et al. Epoprostenol therapy decreases elevated circulating levels of monocyte chemoattractant protein- 1 in patients with primary pulmonary hypertension. Circ J 2004; 68: 227-231.

47 Strassheim D, Riddle SR, Burke DL, et al. Prostacyclin inhibits IFN- $\gamma$-stimulated cytokine expression by reduced recruitment of $\mathrm{CBP} / \mathrm{p} 300$ to STAT1 in a SOCS-1-independent manner. J Immunol 2009; 183: 6981-6988.

48 Shao DD, Suresh R, Vakil V, et al. Pivotal advance: Th- 1 cytokines inhibit, and Th-2 cytokines promote fibrocyte differentiation. J Leukoc Biol 2008; 83: 1323-1333.

49 Mehrad B, Burdick MD, Strieter RM. Fibrocyte CXCR4 regulation as a therapeutic target in pulmonary fibrosis. Int J Biochem Cell Biol 2009; 41: 1708-1718.

50 Sakai N, Wada T, Matsushima K, et al. The renin-angiotensin system contributes to renal fibrosis through regulation of fibrocytes. J Hypertens 2008; 26: 780-790. 